\title{
COMPATIBLE GROUP TOPOLOGIES
}

\author{
KEVIN J. SHARPE
}

ABSTRACT. Two topologies defined on some space are compatible if they contain in common a Hausdorff topology. The following result is proved for two compatible group topologies $a_{1}$ and $a_{2}$. Suppose $a_{1}$ is locally compact and $Q_{2}$ is locally countably compact, and there is a nonvoid $a_{2}$-open set contained in some $a_{1}$-Lindelö set. Then $a_{1} \subseteq Q_{2}$. This result is a stronger version of a theorem by Kasuga, in which two group topologies are shown to be equal if both of them are locally compact and $\sigma$-compact, and they are compatible.

This paper arises from a desire to find sufficient conditions which would make two group topologies on a group comparable. It is a stronger version of a theorem of Kasuga's [3] or [2, p. 58, 6.19] which says that two locally compact $\sigma$-compact group topologies defined on some group are equal if they contain in common a Hausdorff topology. This last restriction on the two topologies I call the compatibility condition: two topologies on some space are compatible if there is a Hausdorff topology contained in common by them. It seems that this condition is the important key by which two topologies can be related.

Interesting questions arise when two topologies are not compatible. It can be shown, for example, that in certain circumstances a group can be decomposed into the product of two subgroups: one on which the two relative topologies are compatible and thus comparable, and the other on which functions continuous in both topologies are constant [4, Chapter 7].

The compatibility condition links two topologies as in the following lemma. An immediate corollary from this is that if one of the two compatible topologies is compact, then it is weaker than the other. Following the lemma is the main result of this paper.

Lemma. If $\mathfrak{Q}_{1}$ and $\mathbb{Q}_{2}$ are two compatible topologies on a space $X$, then any compact subset of either $\left(X, \mathbb{Q}_{1}\right)$ or $\left(X, \mathbb{Q}_{2}\right)$ is both $\mathbb{Q}_{1} \cdot$ and $\mathbb{Q}_{2}$. closed.

Theorem. Let $G$ be a group on which are defined two compatible group topologies $\mathbb{Q}_{1}$ and $\mathbb{Q}_{2},\left(G, \mathbb{Q}_{1}\right)$ being locally compact and $\left(G, \mathbb{A}_{2}\right)$ locally

Received by the editors April 2, 1975.

AMS (MOS) subject classifications (1970). Primary 22D05.

Key words and phrases. Compatible group topologies, comparable group topologies, $\sigma$-compact topological groups. 
countably compact. Then $\mathbb{U}_{1} \subseteq \mathbb{U}_{2}$ if there is a nonempty $\mathbb{G}_{2}$-open set $N$ contained in some $\mathbb{G}_{1}$-Lindelöf set, say $K$.

Proof. Let $A$ be an $\mathbb{Q}_{1}$-open neighbourhood of the identity, and choose $B$ another $\mathscr{G}_{1}$-open neighbourhood of the identity such that $B^{2} \subseteq A$. Because of local compactness and regularity there is an $\mathbb{Q}_{1}$-open symmetric neighbourhood $C$ of the identity with $\mathscr{Q}_{1}$-compact $\mathbb{Q}_{1}$-closure, such that $e \in C=C^{-1}$ $\subseteq C^{-} \subseteq B \subseteq B^{2} \subseteq A$, where, as in the rest of this proof, - denotes $\mathbb{G}_{1}$-closure.

I will show that the identity is in the $\mathbb{G}_{2}$-interior of $A$, and thus every $\mathbb{G}_{1}$-open set is $\mathbb{G}_{2}$-open.

To do this take an $\mathbb{Q}_{2}$-open set $M$ whose $\mathbb{Q}_{2}$-closure $M^{=}$is in $N$, and select a countable cover of $N$, say $\left\{x_{i} C\right\}_{i=1}^{\infty}$, from $\{x C: x \in K\}$. Then $M^{=} \subseteq$ $\bigcup_{i=1}^{\infty} x_{i} C^{-}$. Each $x_{i} C^{-}$is a compact subset of $\left(G, \mathbb{G}_{1}\right)$ and by the above lemma, is a closed subset of $\left(G, \mathbb{G}_{2}\right)$, Thus $M^{=}$is the union of a countable number of $\mathbb{C}_{2}$-closed sets, $\bigcup_{i=1}^{\infty} x_{i} C^{-} \cap M^{=} ; M^{=}$is also a locally countably compact regular space, For some $i$, therefore, $x_{i} C^{-} \cap:^{=}$will have nonvoid $\left.\mathbb{G}_{2}\right|_{V_{1}}=$ interior $[2, \mathrm{p}, 42,5.28]$; that is, there is an $\mathbb{G}_{2}$-open set $E$ such that $E \cap M^{=} \subseteq x_{i} C^{-}$and $E \cap M^{=}$is nonvoid. But then of course $E \cap M$ is also nonvoid, in fact is an $\mathbb{G}_{2}$-open set, and is a subset of $x_{i} C^{-}$. Thus there is an $\mathbb{G}_{2}$-open set $F$ contained in $C^{-}$.

Let $x$ be an element of $F$. Then $e \in x^{-1} F \subseteq x^{-1} C^{-} \subseteq\left(C^{-}\right)^{-1}\left(C^{-}\right)=$ $\left(C^{-}\right)^{2} \subseteq B^{2} \subseteq A$, and $x^{-1} F$ is an $\mathbb{G}_{2}$-open set.

Corollary. If $\mathbb{A}_{1}$ and $\mathbb{Q}_{2}$ are two compatible locally compact group topologies on a group $G$, and $\left(G, \mathbb{G}_{1}\right)$ is $\sigma$-compact, then $\mathbb{A}_{1} \subseteq \mathbb{G}_{2}$.

Lemma 2.6 in Bichteler's paper [1] is interesting. It says that for $\mathfrak{C}_{1}$ and $\mathscr{G}_{2}$ compatible locally compact group topologies for a group $G$, then $\mathscr{G}_{1}$ $=\mathbb{G}_{2}$ if there is in $G$ a $\sigma$-compact subgroup $U$ both $\mathbb{Q}_{1}$ - and $\mathbb{Q}_{2}$-closed, if in the relative topologies $\left(U, \mathbb{Q}_{1}\right)=\left(U, \mathbb{Q}_{2}\right)$, and if in the natural topologies $\left(G / U, \hat{\mathbb{G}}_{1}\right)=\left(G / U, \hat{\mathbb{G}}_{2}\right)$. It is possible to prove something similar using the theorem above, but leaving the topologies comparable rather than equal. Suppose $\mathbb{G}_{1}$ and $\mathbb{Q}_{2}$ are compatible group topologies for $G$, for which $G$ is $\mathbb{A}_{1}$ locally compact and $\mathbb{Q}_{2}$-locally countably compact, and there is a subgroup $U$ of $G$ which is contained in some $\mathbb{Q}_{1}$-Lindelöf set $K_{.}$. Bichteler's condition that the topological groups $\left(G / U, \hat{\mathbb{G}}_{1}\right)$ and $\left(G / U, \hat{\mathbb{G}}_{2}\right)$ be equal (which requires $U$ to be both $\mathscr{G}_{1}$ - and $\mathscr{U}_{2}$-closed), actually boils down to just taking an $\mathbb{G}_{2}$-open set $V^{1}$ and an $\mathbb{G}_{1}$-open neighbourhood $V$ of the identity with $\mathbb{Q}_{1}$-compact $\mathbb{G}_{1}$-closure, such that $V U=V^{1} U$. Now $V^{1} U$ is an $\mathbb{G}_{2}$-open set, and using the fact that $\left(G, \mathbb{Q}_{1}\right)$ is locally compact, there is an $\mathscr{G}_{1}$ - - -compact set $K^{1}$ containing $K$. Then $V^{-} K^{1}$ is $\mathscr{A}_{1}-\sigma$-compact and $V^{1} U \subseteq V^{-} K^{1}$.

Hence $\mathscr{A}_{1} \subseteq \mathscr{A}_{2}$. 
This paper is part of a $\mathrm{Ph}$. $\mathrm{D}$. thesis submitted to La Trobe University, Melbourne, Australia, for which my supervisor was Dr. Graham Elton.

\section{REFERENCES}

1. K. Bichteler, Locally compact topologies on a group and the corresponding continuous irreducible representations, Pacific J. Math. 31 (1969), 583-593. MR 41 \#394.

2. E. Hewitt and K. A. Ross, Abstract harmonic analysis. Vol I: Structure of topological groups. Integration theory, group representations, Die Grundlehren der math. Wissenschaften, Band 115, Academic Press, New York; Springer-Verlag, Berlin, 1963. MR $28 \# 158$.

3. T. Kasuga, On the isomorphism of topological groups, Proc. Japan Acad. 29 (1953), 435-438. MR 16, 112.

4. K. J. Sharpe, Relationships between group topologies, Ph. D. Thesis, La Trobe University, Melbourne, 1974 (unpublished).

DEPARTMENT OF MATHEMATICS, LA TROBE UNIVERSITY, MELBOURNE, AUSTRALIA

Current address: Episcopal Divinity School, Cambridge, Massachusetts 02138 